\title{
Molecular Weight Dependence of Relaxation Time Spectra for the Entanglement and Flow Behavior of Monodisperse Linear Flexible Polymers
}

\author{
J. K. Jackson, ${ }^{\dagger}$ M. E. De Rosa, ${ }^{\ddagger}$ and H. H. Winter ${ }^{*},+, \ddagger$ \\ Departments of Chemical Engineering and Polymer Science and Engineering, \\ University of Massachusetts, Amherst, Massachusetts 01003
}

Received October 11, 1993; Revised Manuscript Received February 2, $1994^{\circ}$

\begin{abstract}
A simple model is proposed for the molecular weight dependence of the relaxation time spectrum of linear flexible polymers with molecules of nearly uniform length. A systematic approach for determining the parameters of the relaxation time spectrum from dynamic mechanical data, $G^{\prime}(\omega)$ and $G^{\prime \prime}(\omega)$, shows the possibilities and the limits of this model. The necessary parameters are $n_{\mathrm{e}}, G_{\mathrm{N}}{ }^{\circ}$, and $\lambda_{\max }$ for the entanglement and flow behavior and $\lambda_{c}$ and $n_{g}$ for the crossover to the high-frequency glass behavior. Generic values are proposed for polystyrene and polybutadiene of high molecular weight.
\end{abstract}

\section{Introduction}

The linear viscoelastic properties of a polymer with long linear flexible chains of (nearly) uniform length are known to follow a unique pattern. ${ }^{1-5}$ Chemical details of macromolecules are important for the short-time behavior but have less significance at longer time scales. It is generally agreed that the entanglement and flow behavior of linear macromolecules can be modeled independently of chain chemistry. It therefore is expected that such materials are characterized by a unique relaxation time spectrum, $H(\lambda)$. Molecular dynamics theories predict the spectrum from models for the molecular motion and the averged forces on the molecules. ${ }^{6-11}$ Two well-known predictions for $H(\lambda)$ are the spectrum of Rouse, ${ }^{6}$ who proposed a beadspring model which scales as $\lambda^{-1 / 2}$, and the spectrum which Doi $^{7}$ derived from the reptation theory of de Gennes ${ }^{8}$ which scales as $\lambda^{+1 / 2}$. These theories provide insight into the meaning of macroscopically measured material functions and the associated parameters such as plateau modulus, $G_{N^{\circ}}$, and longest relaxation time, $\lambda_{\max }$, but lack quantitative agreement with experimental data such as the dynamic moduli, $G^{\prime}(\omega)$ and $G^{\prime \prime}(\omega)$.

Experimentalists have also made substantial progress toward finding the relaxation spectrum of linear flexible macromolecules. Tobolsky ${ }^{12}$ proposed an empirical model for the relaxation spectrum based on stress relaxation data. He suggested that a three-parameter model describes the relaxation modulus, $G(t)$, and then inferred a box-wedge shape for $H(\lambda)$ in terms of the three parameters. While the "box" regions could not be confirmed experimentally, the "wedge" region seems to describe the high-frequency glass behavior. Ferry ${ }^{13}$ reviewed several other empirical methods of approximating $H(\lambda)$ from rheological data. These methods involve not only the absolute values of material functions but also their derivatives. This makes them very sensitive to scatter in the input data. Researchers have also investigated the merits of discrete relaxation time spectra. A review of current methods of evaluating discrete relaxation spectra from dynamic mechanical data has been presented by Orbey and Dealy. ${ }^{14}$

The relaxation time spectrum cannot be measured directly but rather must be evaluated from observable material functions such as the storage modulus, $G^{\prime}(\omega)$,

\footnotetext{
Department of Chemical Engineering.

\$ Department of Polymer Science and Engineering.

- Abstract published in Advance ACS Abstracts, March 15, 1994.
}

and the loss modulus, $G^{\prime \prime}(\omega)$

$$
\begin{aligned}
& G^{\prime}(\omega)=\int_{0}^{\lambda_{\max }} H(\lambda) \frac{\omega^{2} \lambda^{2}}{1+\omega^{2} \lambda^{2}} \frac{\mathrm{d} \lambda}{\lambda} \\
& G^{\prime \prime}(\omega)=\int_{0}^{\lambda_{\max } H(\lambda) \frac{\omega \lambda}{1+\omega^{2} \lambda^{2}} \frac{\mathrm{d} \lambda}{\lambda}}
\end{aligned}
$$

With an appropriate representation of $H(\lambda)$, one can model the dynamic moduli as well as other material functions such as the relaxation modulus, $G(t)$, or the dynamic viscosity, $\eta^{*}(\omega)$.

A relaxation time spectrum consisting of a superposition of two power laws ${ }^{5}$ has been shown to represent the linear viscoelastic behavior of monodisperse linear flexible polymers in the flow and entanglement regime. We propose to evaluate the parameters associated with such a spectrum (BSW spectrum) for the cases of polystyrene and polybutadiene. Previous work ${ }^{5,15}$ focused on obtaining the best approximation of the data for individual samples of different molecular weights. In this study, we outline a method of obtaining a single set of parameters which best approximates the entire range of data for all of the molecular weights above the critical molecular weight which is required for forming physical entanglements. In the process, we clarify the features of the spectrum which are common to all linear flexible polymers and interpret the physical significance of the parameters.

\section{The BSW Spectrum and Its Properties}

The BSW spectrum ${ }^{5}$ for linear flexible polymers with molecules of (nearly) uniform length may be written as

$$
H(\lambda)= \begin{cases}n_{\mathrm{e}} G_{\mathrm{N}} \circ\left[\left(\frac{\lambda}{\lambda_{\mathrm{c}}}\right)^{-n_{\mathrm{g}}}+\left(\frac{\lambda}{\lambda_{\max }}\right)^{n_{\mathrm{e}}}\right], & \text { for } \lambda \leq \lambda_{\max } \\ 0, & \text { for } \lambda>\lambda_{\max }\end{cases}
$$

where $G_{N}{ }^{\circ}$ is the plateau modulus, $\lambda_{\max }$ is the longest relaxation time of the material, $n_{\mathrm{e}}$ and $n_{\mathrm{g}}$ are the slopes of the spectrum in the entanglement and high-frequency glass transition zones, respectively, and $\lambda_{c}$ is the crossover time to the glass transition. The first term in the brackets stands for the high-frequency glass transition region as proposed by Tobolsky. ${ }^{12}$ The second term describes the self-similar relaxation in the entanglement region. The cutoff at $\lambda_{\max }$ is the most simple way of introducing the long-time behavior. A schematic of the BSW spectrum and its parameters is presented in Figure 1. 


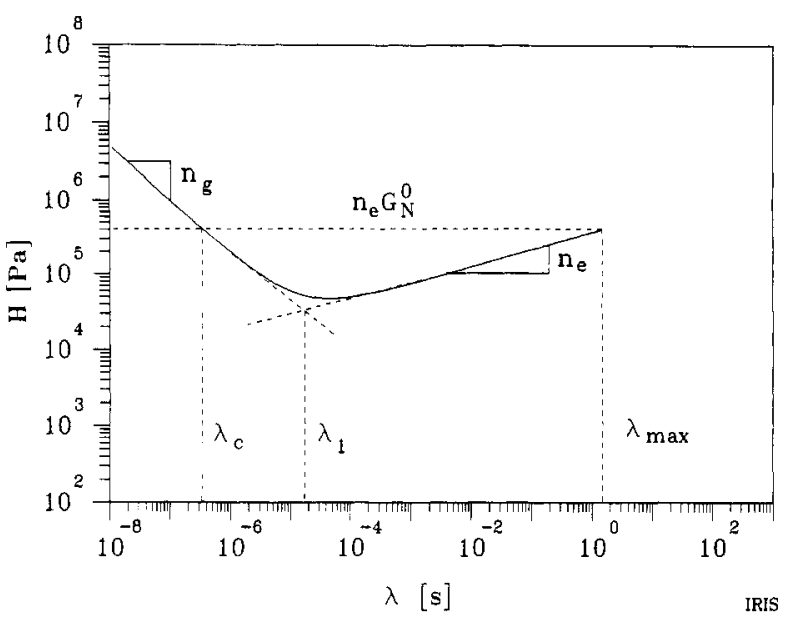

Figure 1. Schematic of the BSW model for the relaxation spectrum of monodisperse linear flexible polymer. The five parameters that define the spectrum are the glass transition slope $\left(n_{\mathrm{g}}\right)$, the entanglement slope $\left(n_{\mathrm{o}}\right)$, the plateau modulus $\left(G_{\mathrm{N}}{ }^{\circ}\right)$, the crossover relaxation time $\left(\lambda_{c}\right)$, and the longest relaxation time $\left(\lambda_{\max }\right)$.

Using the BSW spectrum and neglecting the glass transition, it is a simple matter to calculate other viscoelastic properties of interest such as zero-shear viscosity, $\eta_{0}$

$$
\eta_{0}=\int_{0}^{\lambda_{\max }} H(\lambda) \mathrm{d} \lambda=\frac{n_{\mathrm{e}}}{n_{\mathrm{e}}+1} G_{\mathrm{N}}{ }^{\circ} \lambda_{\max }
$$

or the recoverable compliance, $J_{\ominus}^{\circ}$,

$$
J_{\mathrm{e}}^{\circ}=\frac{1}{\eta_{0}^{2}} \int_{0}^{\lambda_{\max }} H(\lambda) \lambda \mathrm{d} \lambda=\frac{1}{G_{\mathrm{N}}}\left(1+\frac{1}{n_{\mathrm{e}}^{2}+2 n_{\mathrm{e}}}\right)
$$

Another important property of the BSW spectrum is its prediction of the high-frequency asymptote of $G^{\prime \prime}(\omega)$ when the glass transition is neglected. We see from eq 2

$$
\begin{aligned}
\lim _{\omega \rightarrow \infty} G^{\prime \prime}(\omega) & =\lim _{\omega \rightarrow \infty} \int_{0}^{\lambda_{\max }} n_{\mathrm{e}} G_{\mathrm{N}} \circ\left(\frac{\lambda}{\lambda_{\max }}\right)^{n_{0}} \frac{\omega \mathrm{d} \lambda}{1+(\omega \lambda)^{2}} \\
& =G_{\mathrm{N}} \circ \frac{n_{\mathrm{e}} \pi / 2}{\cos \left(n_{\mathrm{e}} \pi / 2\right)}\left(\omega \lambda_{\max }\right)^{-n_{\mathrm{e}}}
\end{aligned}
$$

Thus the limiting slope of $G^{\prime \prime}(\omega)$ gives the same exponent value as the slope of the entanglement region of the BSW spectrum, $n_{\mathrm{e}}$. The parameter $n_{\mathrm{e}}$ is determined initially from the slope of $G^{\prime \prime}(\omega)$ in the entanglement regime. Though this is a close approximation, it is only precise in the limiting case of eq 6 . Thus it is necessary to iterate on $n_{\mathrm{e}}$ to obtain a value consistent with eq 4.

Molecular Weight Dependence of BSW Parameters. Of the BSW parameters, $n_{\mathrm{e}}, G_{\mathrm{N}}{ }^{\circ}, n_{\mathrm{g}}, \lambda_{\mathrm{c}}$, and $\lambda_{\max }$, only $\lambda_{\max }$ is assumed to have molecular weight dependence. The ratio $\lambda_{\max } / \lambda_{c}$ denotes the width of the entanglement plateau. We propose that the longest time, $\lambda_{\max }$, scales as

$$
\lambda_{\max }=\lambda_{c}\left(\frac{M}{M_{c}}\right)^{z}
$$

where the parameters $\lambda_{c}$ and $M_{c}$ are crossover relaxation time and molecular weight, respectively. The exponent $z$ has a value of $\sim 3.3-3.5$ as reported by many researchers. ${ }^{16,17}$ The molecular weight, $M$, is almost always replaced by the weight-average molecular weight, $M_{w}$, since no truly monodisperse materials are available. Equation 7 can be
Table 1. Molecular Weights of Polystyrene

\begin{tabular}{crc}
\hline sample & \multicolumn{1}{c}{$M_{w}$} & $M_{w} / M_{n}$ \\
\hline PS-1 & 34000 & 1.05 \\
PS-2 & 65000 & 1.02 \\
PS-3 & 125000 & 1.05 \\
PS-4 & 292000 & 1.09 \\
PS-5 & 757000 & 1.09 \\
PS-6 & 2540000 & 1.13
\end{tabular}

substituted into eq 3 to obtain

$$
\begin{aligned}
H(\lambda)=n_{\mathrm{e}} G_{\mathrm{N}}\left[\left(\frac{\lambda}{\lambda_{\mathrm{c}}}\right)^{-n_{\mathrm{c}}}+\left(\frac{\lambda}{\lambda_{\mathrm{c}}}\left(\frac{M_{\mathrm{c}}}{M}\right)^{2}\right)^{n_{\mathrm{e}}}\right], & \\
& \text { for } \lambda \leq \lambda_{\mathrm{c}}\left(\frac{M}{M_{\mathrm{c}}}\right)
\end{aligned}
$$

This formulation shows explicitly the parameters necessary to describe monodisperse linear flexible polymers above the entanglement molecular weight.

Equation 7 for $\lambda_{\max }$ can be substituted into eq 4 and combined with the well-established scaling of $\eta_{0}$ with molecular weight ${ }^{16}$

$$
\eta_{0}=a M_{\mathrm{w}}^{2}
$$

to obtain an expression for the prefactor, $a$, in terms of BSW parameters,

$$
\eta_{0}=\frac{n_{\theta}}{n_{\theta}+1} G_{N}{ }^{\circ} \lambda_{c}\left(\frac{M_{\mathrm{w}}}{M_{\mathrm{c}}}\right)^{z}
$$

The characteristic time, $\lambda_{1}$, at which the asymptotes of the entanglement region and the glass transition region intersect (Figure 1) could be chosen as a reference time instead of $\lambda_{c}$. While either time is acceptable, the advantage of using $\lambda_{c}$ is that it does not change with molecular weight as $\lambda_{1}$ does. The reference time, $\lambda_{1}$, is related in a straightforward manner to $\lambda_{c}$ and $M_{c}$. Given the definition of the BSW spectrum for the entanglement and glass transition regions, it is clear that the two power laws intersect at $\lambda=\lambda_{1}$ :

$$
n_{\mathrm{e}} G_{\mathrm{N}} \cdot\left(\frac{\lambda_{1}}{\lambda_{\max }}\right)^{n_{\mathrm{e}}}=n_{\mathrm{e}} G_{\mathrm{N}} \circ\left(\frac{\lambda_{1}}{\lambda_{\mathrm{c}}}\right)^{-n_{\mathrm{g}}}
$$

Rearrangement of eq 11 and introduction of eq 7 give

$$
\lambda_{1}=\lambda_{\mathrm{c}}\left(\frac{M}{M_{\mathrm{c}}}\right)^{3.4 n_{\mathrm{d}}\left(n_{\mathrm{e}}+n_{\mathrm{g}}\right)}
$$

Therefore, as $M \rightarrow M_{c}$, the times $\lambda_{c}$ and $\lambda_{1}$ coincide. The exponent has a value close to unity when using the parameter values below. Whether one chooses to use $\lambda_{c}$ or $\lambda_{1}$ as the reference time, the choice simply fixes the spectrum at a reference point and does not suggest anything about the physics of the glassy relaxation behavior, which is outside the scope of this paper.

\section{Experimental Section}

For this study, we chose dynamic mechanical data from six nearly monodisperse polystyrenes previously published by Schauberger.4 Details of the experimental method can be found in the original publication. Table 1 lists the molecular weights and $M_{w} / M_{\mathrm{n}}$ ratios of these samples as detemined by gel permeation chromatography (GPC). The dynamic moduli are displayed in Figures 2 and 3.

In addition, dynamic moduli were measured for seven nearly monodisperse polybutadienes with similar cis, trans, and vinyl content. The details of the synthesis and rheological measurements are given in a previous paper. ${ }^{15}$ The molecular weight 


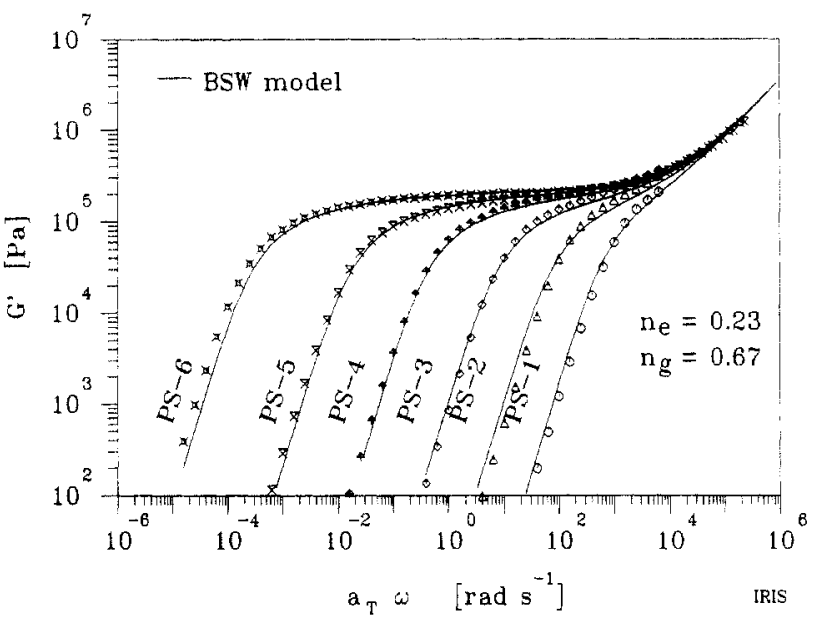

Figure 2. Storage modulus, $G^{\prime}$, of six nearly monodisperse polystyrenes. The molecular weights and BSW parameters are given in Tables 1 and 4, respectively. The measurements were performed at $180^{\circ} \mathrm{C}$. The line through the data represents the BSW model (eq 3).

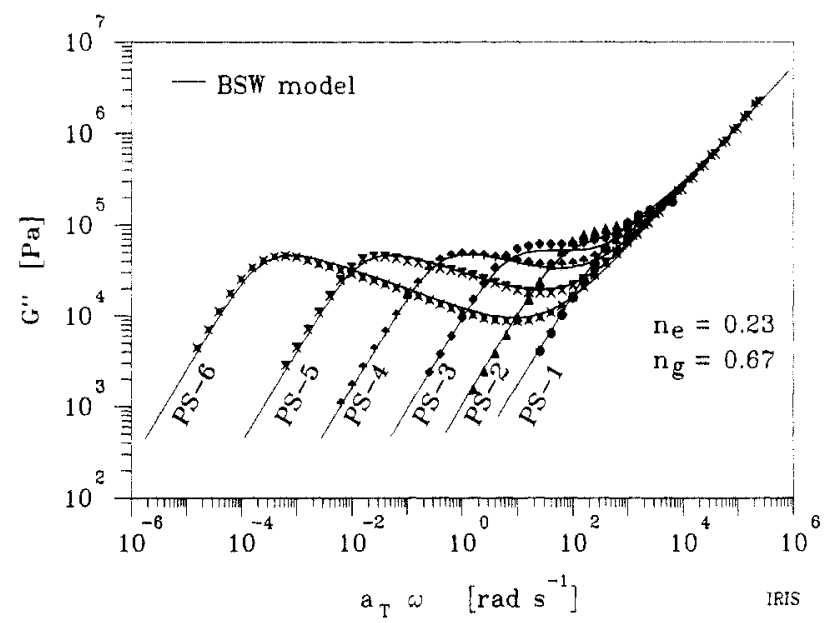

Figure 3. Loss modulus, $G^{\prime \prime}$, of six nearly monodisperse polystyrenes. The molecular weights and BSW parameters are given in Tables 1 and 4 , respectively. The measurements were performed at $180^{\circ} \mathrm{C}$. The line through the data represents the BSW model (eq 3).

Table 2. Molecular Weights of Polybutadiene

\begin{tabular}{ccc}
\hline sample & $M_{\mathrm{w}}$ & $M_{\mathrm{w}} / M_{\mathrm{n}}$ \\
\hline PBD-18 & 18100 & 1.05 \\
PBD-20 & 20700 & 1.04 \\
PBD-38 & 37900 & 1.05 \\
PBD-44 & 44100 & 1.04 \\
PBD-70 & 70200 & 1.02 \\
PBD-97 & 97000 & 1.07 \\
PBD-201 & 201000 & 1.27
\end{tabular}

Table 3. Chemistry of Polybutadiene

\begin{tabular}{ccccc}
\hline sample & \% cis & \% trans & \% vinyl & $T_{\mathrm{z}}\left({ }^{\circ} \mathrm{C}\right)$ \\
\hline PBD18 & 41 & 52 & 7.30 & -92 \\
PBD20 & 39 & 53 & 8.19 & -91 \\
PBD38 & 41 & 51 & 7.70 & -92 \\
PBD44 & 39 & 53 & 8.11 & -90 \\
PBD70 & 43 & 50 & 7.30 & -93 \\
PBD97 & 41 & 51 & 8.04 & -90 \\
PBD201 & 44 & 48 & 7.88 & -92
\end{tabular}

data are given in Table 2 , and the microstructure data are given in Table 3. The dynamic moduli are shown in Figure 4 and 5.

\section{Parameter Evaluation}

1. Parameter Initialization. Before one can begin an iteration, initial values of each parameter must be chosen.
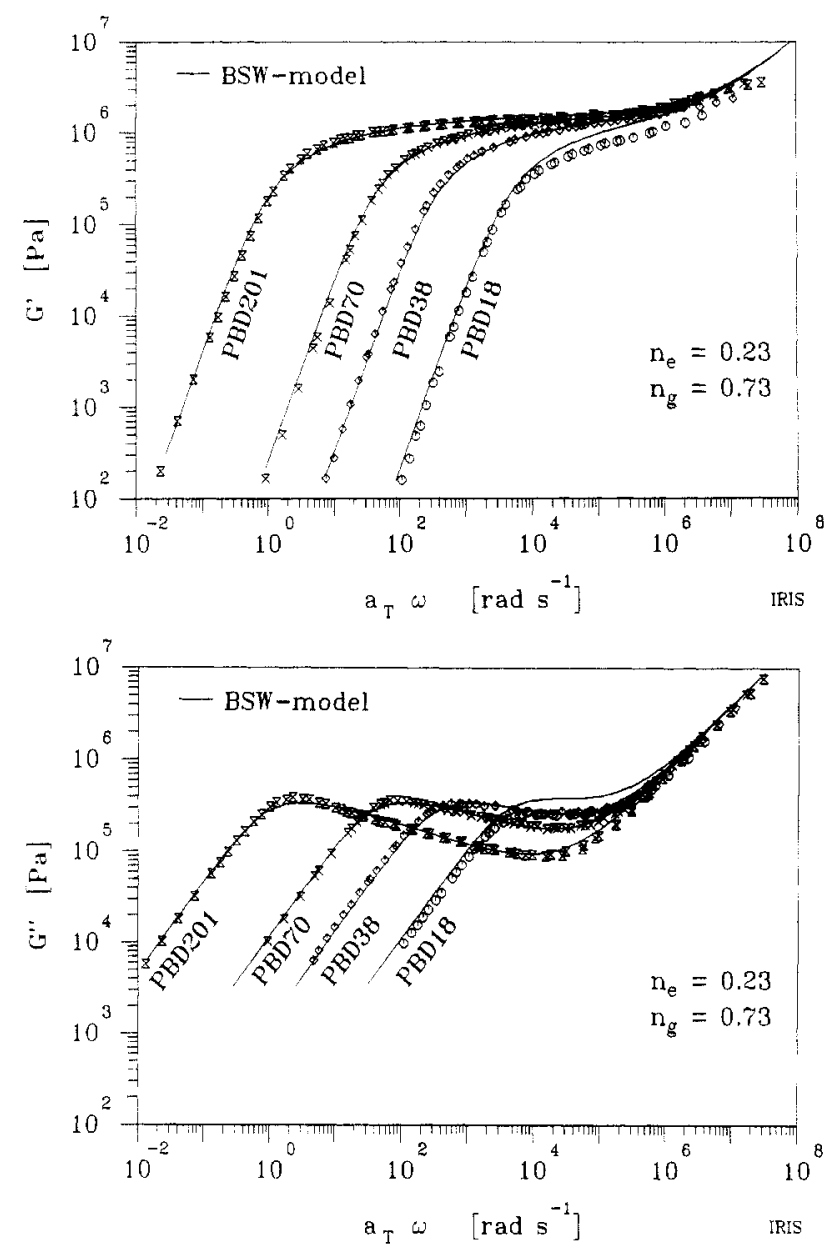

Figure 4. (a) Storage modulus $\left(G^{\prime}\right)$ and (b) loss modulus $\left(G^{\prime \prime}\right)$ of four nearly monodisperse polybutadienes. The molecular weights and BSW parameters are given in Tables 2 and 4 , respectively. The measurements were performed at $28^{\circ} \mathrm{C}$. The line through the data represents the BSW model (eq 3).

The Scaling Exponent, z. The zero-shear viscosities from the dynamic data for each sample are plotted versus $M_{\mathrm{w}}$ and fit with a power law as shown in Figure 6. The power law exponent is the initial value of the exponent $z$ for the molecular weight dependence.

BSW Slopes, $n_{e}$ and $n_{k}$. The slopes of the glass transition and entanglement power laws of the BSW spectrum are evaluated next. For that purpose a line is fit to the entanglement region of the loss modulus, $G^{\prime \prime}$. Its slope gives an approximate value for $n_{e}$. Then a line is fit to the glass transition region of $G^{\prime \prime}$ to obtain an approximate slope $n_{\mathrm{g}}$ (see Figure 7). The most accurate evaluation of slopes is possible from the highest molecular weight, which has the most pronounced entanglement behavior. This can be seen in Figure 8, where $G^{\prime \prime}$ curves of several molecular weights of polybutadiene are scaled so that the entanglement region overlays. Also included is the asymptote as calculated from eq 6 . It is clear that even the highest molecular weight does not exactly match the slope of the asymptote.

The Plateau Modulus, $G_{\mathrm{N}}{ }^{\circ}$. The initial estimate of $G_{\mathrm{N}}{ }^{\circ}$ is chosen to fix the entanglement power law on the $y$-axis such that the plateau region of $G^{\prime}(\omega)$ matches the simulation for the data of the highest molecular weight sample.

The Longest Relaxation Time, $\lambda_{\max }$, and the Crossover Relaxation Time, $\lambda_{c}$. Once the plateau modulus has been chosen, it is possible to obtain $\lambda_{\max }$ by fixing the location of the entanglement power law on the $x$-axis such that the terminal zone of the dynamic moduli data match 

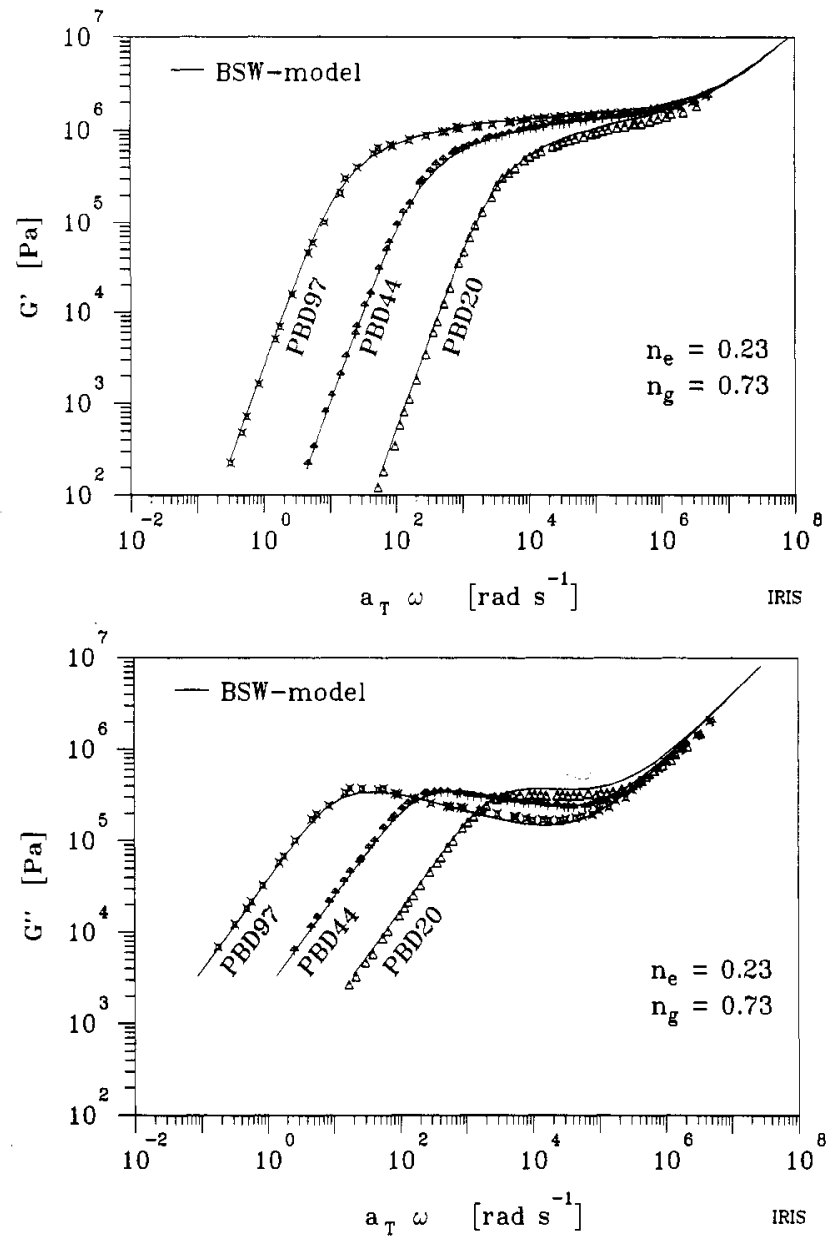

Figure 5. (a) Storage modulus $\left(G^{\prime}\right)$ and (b) loss modulus $\left(G^{\prime \prime}\right)$ of three nearly monodisperse polybutadienes. The molecular weights and BSW parameters are given in Tables 2 and 4, respectively. The measurements were performed at $28^{\circ} \mathrm{C}$. The line through the data represents the BSW model (eq 3 ).

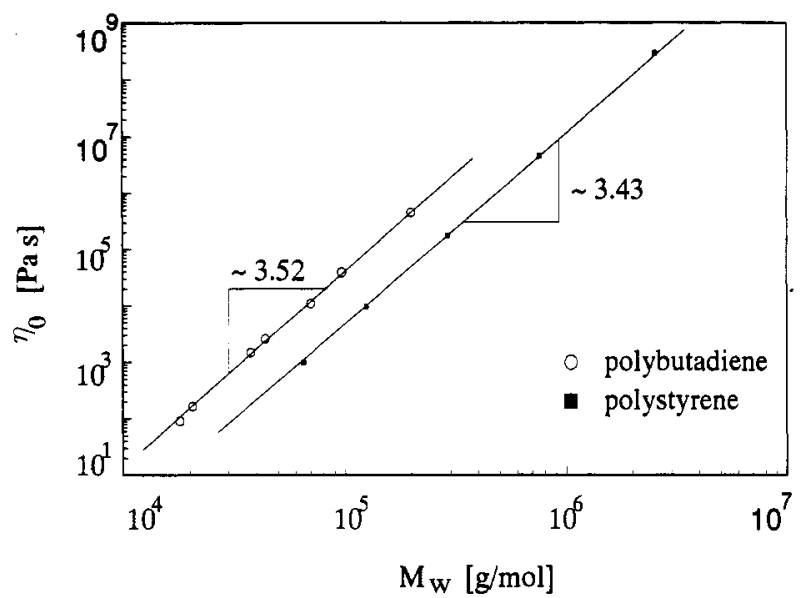

Figure 6. Scaling of zero-shear viscosity with molecular weight for polybutadiene and polystyrene. The polystyrene and polybutadiene have slopes of 3.43 and 3.52 , respectively. The reference temperature for polystyrene is $180^{\circ} \mathrm{C}$ and the reference temperature of polybutadiene is $28^{\circ} \mathrm{C}$.

the simulation. The crossover time, $\lambda_{c}$, is obtained by fixing the glass transition power law on the $x$-axis such that the glass transition zone of the dynamic moduli data match the simulation. Both of these parameters should be evaluated for each individual molecular weight sample. A simple rearrangement of eq 7 allows for calculation of $M_{\mathrm{c}}$ once $\lambda_{\max }$ and $\lambda_{\mathrm{c}}$ are known.

2. Iteration Procedure for Improving the Fit. One substitutes the initial values obtained in step 1 into eq 3
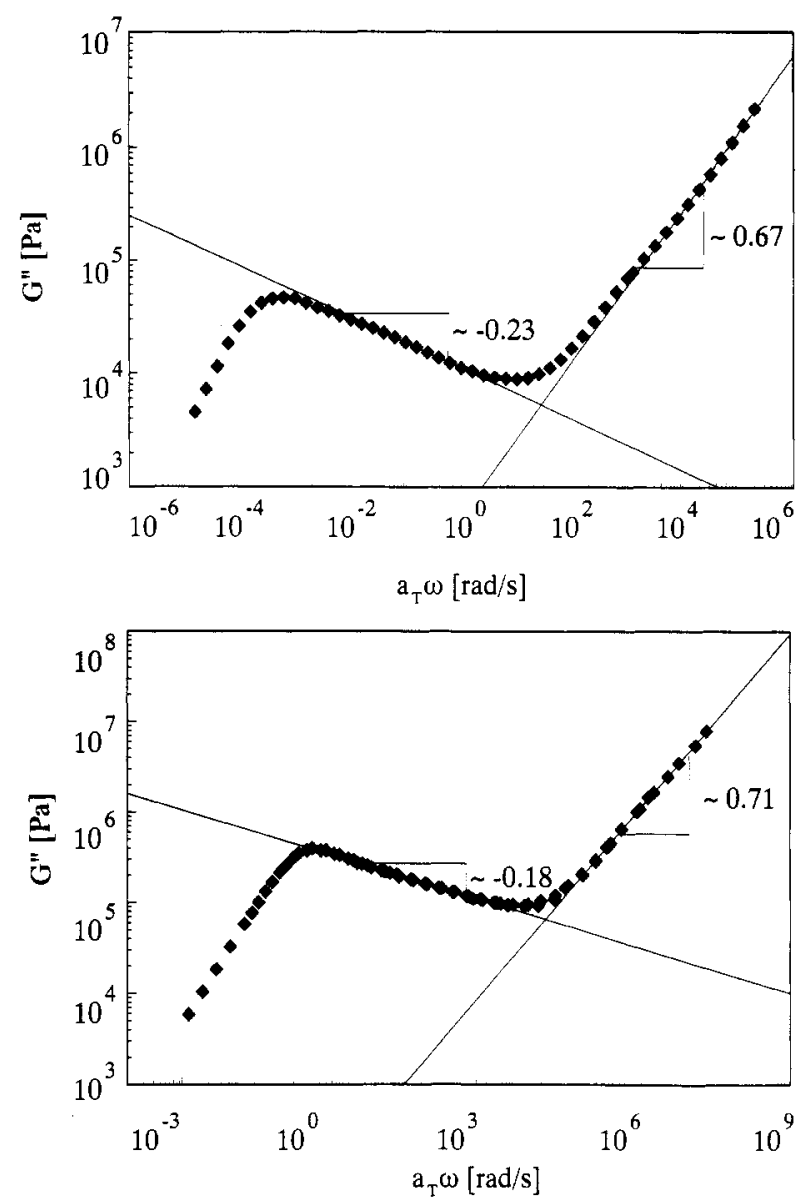

Figure 7. (a) Slopes of the entanglement zone and the glass transition zone of polystyrene with molecular weight of 2540000 . These values were used as initial slope values for the relaxation time spectrum. The reference temperature is $180^{\circ} \mathrm{C}$. (b) Slopes of the entanglement zone and the glass transition zone of polybutadiene with molecular weight of 201000 . These values were used as initial slope values for the relaxation time spectrum. The reference temperature is $28^{\circ} \mathrm{C}$.

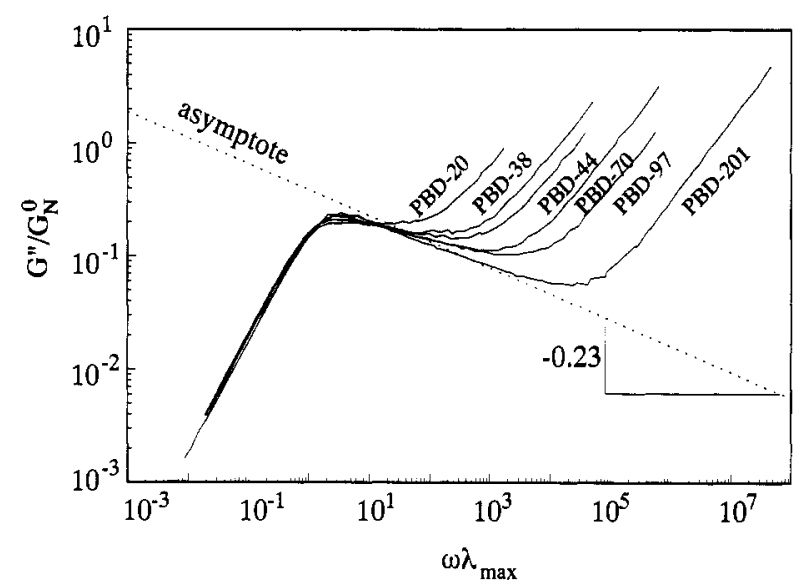

Figure 8. Comparison of entanglement slopes for polybutadienes with different molecular weights. The actual value used for the entanglement slope is represented by the asymptote (dashed line) defined by eq 6 .

and calculates the dynamic moduli from eqs 1 and 2. Iteration on each parameter improves the agreement of the $G^{\prime}(\omega)$ and $G^{\prime \prime}(\omega)$ simulation with the dynamic data.

3. Final Parameter Evaluation. The parameters $\lambda_{c}$ and $M_{\mathrm{c}}$ are averaged over all the molecular weights to get final values. It is the averaged values of $\lambda_{c}$ and $M_{c}$ which are used along with $z, n_{\mathrm{e}}, n_{\mathrm{g}}, G_{\mathrm{N}^{\circ}}$, and $M$ to perform the final simulation. 


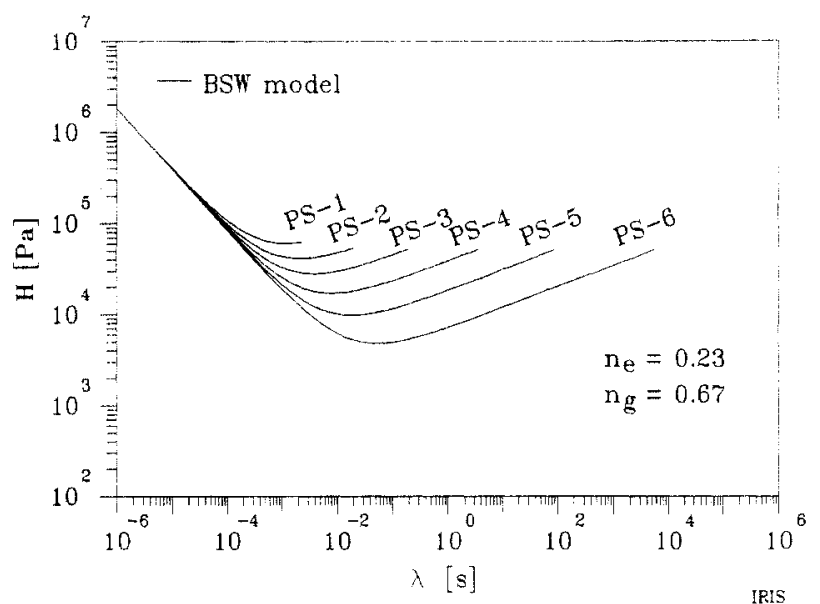

Figure 9. Relaxation time spectrum of six nearly monodisperse polystyrenes. The molecular weights and BSW parameters are given in Tables 1 and 4, respectively. The lines represent the BSW model (eq 3).

Table 4. BSW Parameters of Polybutadiene at $28^{\circ} \mathrm{C}$ and Polystyrene at $180^{\circ} \mathrm{C}$

\begin{tabular}{lll}
\hline parameter & \multicolumn{1}{c}{ PS } & \multicolumn{1}{c}{ PBD } \\
\hline$G_{\mathrm{N}}^{0}(\mathrm{~Pa})$ & 228000 & 1650000 \\
$n_{\mathrm{e}}$ & 0.23 & 0.23 \\
$n_{\mathrm{g}}$ & 0.67 & 0.73 \\
$\lambda_{\mathrm{c}}(\mathrm{s})$ & $2.00 \times 10^{-4}$ & $4.04 \times 10^{-7}$ \\
$M_{\mathrm{c}}$ & 16600 & 2714 \\
$z$ & 3.43 & 3.52 \\
$C_{1}$ & 5.02 & 4.17 \\
$C_{2}(\mathrm{~K})$ & 125.3 & 196.8 \\
$T_{\text {ref }}\left({ }^{\circ} \mathrm{C}\right)$ & 180 & 28
\end{tabular}

\section{Modeling Results}

Using the modeling procedure outlined above, we obtained the spectra parameters for polystyrene and polybutadiene. The spectra evaluated for polystyrene from the dynamic data in Figure 2 and 3 are displayed in Figure 9. The corresponding BSW parameters are listed in Table 4. Time-temperature superposition was performed and the shift factors, $a_{T}$, were presumed to fit the WLF equation

$$
\log a_{T}=\frac{-C_{1}\left(T-T_{\mathrm{ref}}\right)}{C_{2}+\left(T-T_{\mathrm{ref}}\right)}
$$

where $C_{1}$ and $C_{2}$ are the WLF parameters at the reference temperature, $T_{\text {ref. }}$ The WLF parameters from timetemperature superposition are also included in Table 4. The lines in Figures 2 and 3 represent the dynamic moduli calculated from the spectrum displayed in Figure 9.

The spectra evaluated for polybutadiene from the dynamic data in Figures 4 and 5 are displayed in Figure 10. The corresponding BSW parameters as well as the WLF parameters from time-temperature superposition are listed in Table 4. The lines in Figures 4 and 5 represent the dynamic moduli calculated from the spectrum in Figure 10.

\section{Discussion}

The results (Figures 2-5) clearly show that the dynamic moduli of nearly monodisperse polystyrene and polybutadiene are well described by the spectra proposed (Figures 9 and 10 ) over a range of molecular weights. While these results are encouraging, it would be useful to analyze additional data on nearly monodisperse linear flexible polymers with different chemistry and to determine the range of parameter values for different materials. It is
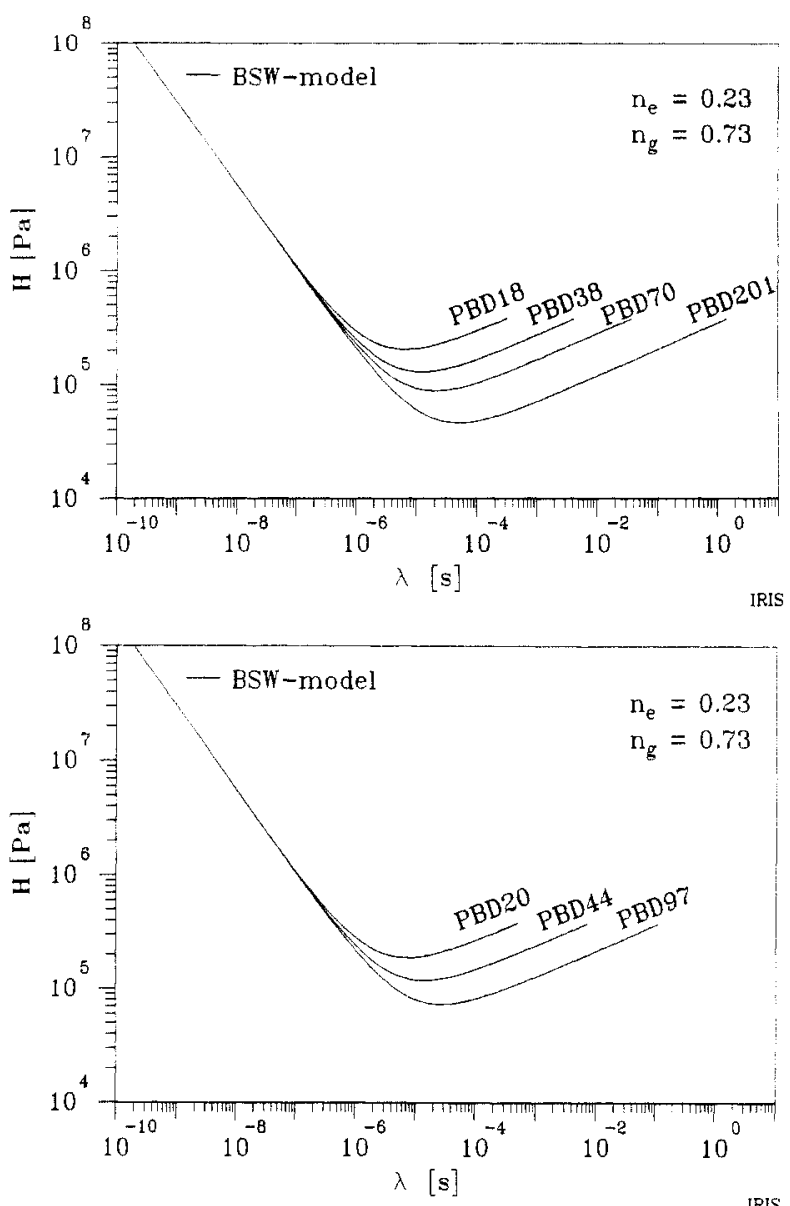

Figure 10. Relaxation time spectra of (a) four nearly monodisperse polybutadienes and (b) three nearly monodisperse polybutadienes. The molecular weights and BSW parameters are given in Tables 2 and 4, respectively. The line through the data represents the BSW model (eq 3 ).

interesting that the slope $n_{\mathrm{e}}$ is the same for polybutadiene and polystyrene, and it would be useful to know whether this is a universal value. A possible explanation of this phenomenon might be contour length fluctuation (Doi $\left.{ }^{18}\right)$.

One source of discrepancies between the data and the predicted moduli come from the scaling exponent, $z$. As described previously, $z$ was obtained from a plot of $\eta_{0}$ versus $M_{\mathrm{w}}$ for all of samples in this study. However, the lower molecular weight samples of polybutadiene and polystyrene may not scale with a 3.4 power but rather with a power between 1 and 3.4 If this is the case, then the lower molecular weights should not be included in the modeling, and $z$ should be obtained from the higher molecular weight samples only.

A second source of discrepancies may be the slight polydispersity of the samples used in this study. While the BSW spectrum proposed for a monodisperse polymer has a sharp cutoff at $\lambda_{\max }$, the actual spectrum for a nearly monodisperse polymer may have a gradual transition to zero near $\lambda_{\max }$. One approach to understanding polydispersity effects is to examine dynamic data on binary blends of different molecular weights. If there is a significant broadening of the loss modulus peak, then one must consider what effect the nearly monodisperse nature of the blend components has on the dynamic data. One could also use the spectrum proposed here to represent the behavior of a single molecular weight and then propose a superposition of the spectra for different molecular weights to describe a molecular weight distribution.

In the case of polybutadiene, an additional problem is the different chemistry for samples of different molecular 
weights. This may be responsible for the low plateau moduli of PBD-18 and PBD-20.

We previously introduced the parameters $M_{\mathrm{c}}$ and $\lambda_{\mathrm{c}}$ to describe the relaxation behavior of linear flexible monodisperse polymers. By presenting these parameters in eq 8 , it was possible to construct a unique expression for the prefactor in the relation $\eta_{0}=a M_{\mathrm{w}}{ }^{2}$ in terms of these two parameters. Their physical meaning is subject to interpretation, but one suggestion is that they correspond to the molecular weight at which entanglement begins. The definition of $M_{\mathrm{c}}$ as used in this analysis of $G^{\prime}(\omega)$ and $G^{\prime \prime}(\omega)$ data differs from the definition of $M_{\mathrm{c}}$ from viscosity data (see data compiled in Ferry ${ }^{13}$ ). Accordingly, their values are slightly different.

The modeling emphasizes the high molecular weight behavior, $M \gg M_{\mathrm{c}}$. Significant deviations were found for the samples with molecular weight in the crossover region, $M \approx M_{\mathrm{c}}$. Our model seems to be too simple for capturing the details of the intermediate behavior of polymers near $M_{c}$. Similar complications are known to occur with the molecular weight dependence of the shear viscosity in the crossover region. The rheological behavior near $M_{\mathrm{c}}$ should be studied further. This study can only be used as a firstorder approximation in this region.

We propose that there exists a generic set of BSW parameters for each chemical species of linear polymer chains. Parameter values have been determined for polystyrene and a specific group of polybutadienes, but such values obviously rely on the accuracy of the data, which is difficult to assess. Variations of the systematic error in the measurement at different laboratories will result in systematic variations of the BSW parameters. The above framework will help to improve and to screen the various experimental methods so that parameter values will eventually consolidate.

\section{Conclusions}

A relaxation time spectrum with a simple power law form achieves good agreement with the dynamic data of nearly monodisperse polystyrene and polybutadiene. A single set of parameters can be evaluated in a consistent manner and used to describe the linear viscoelasticity of a range of molecular weights. More data have to be analyzed in this fashion to obtain generic parameter sets for a wide range of polymers.

A consistent procedure for evaluating the parameters $n_{e}, n_{\mathrm{g}}, G_{\mathrm{N}}{ }^{\circ}, \lambda_{\max }$, and $\lambda_{c}$ has been outlined. Initial values are estimated, iterated to improve the fit, and then averaged over all molecular weights to get final parameter values. The initial values of parameters were all obtained directly from the dynamic data.

There is a natural way of defining and evaluating the crossover parameters $M_{\mathrm{c}}$ and $\lambda_{\mathrm{c}}$ from $G^{\prime}(\omega)$ and $G^{\prime \prime}(\omega)$ data. These parameters are slightly different from those obtained from the crossover in viscosity-molecular weight data.
The molecular weight dependence of the relaxation spectrum is encapsulated in the 3.4 scaling of $\lambda_{\max }$ to $M_{\mathrm{w}}$. The entanglement power law becomes longer with increasing molecular weight while the glass transition power law remains unchanged. This representation of the spectrum and its molecular weight dependence leads naturally to an expression for the prefactor in the viscositymolecular weight scaling relation in terms of the BSW parameters, $z, n_{e}, G_{N^{\circ}}, M_{c}$, and $\lambda_{c}$.

The BSW spectrum may be used as a starting point for the investigation of the behavior of polydisperse and branched flexible polymers. The effect of polydispersity may be considered by studying binary blends of different molecular weights. A mixing rule may be developed based on knowledge of the blend behavior and the behavior of the monodisperse constituents of the blend. Branching may be considered as a deviation from the behavior of the linear chain. While the BSW spectrum comprises part of the relaxation behavior of a branched species, it must be modified to consider the constraints to relaxation imposed by branching. Finally, the BSW spectrum is a simple description of the relaxation of linear flexible polymers to which theories of entanglement relaxation can be compared.

Acknowledgment. This work was supported by the Center for the University of Massachusetts Industry Research in Polymers and by the General Electric Co. We thank Dr. Joe Machado and Dr. Mike Masse at Shell Development $\mathrm{Co}$. for providing us with the polybutadiene samples and characterization data. We also thank Professor Shaw Ling Hsu for the use of his DSC and FT Raman instruments.

\section{References and Notes}

(1) Onogi, S.; Masuda, T.; Kitigawa, K. Macromolecules 1970, 3 , 109.

(2) Graessley, W. Adv. Polym. Sci. 1974, 16.

(3) Marin, G.; Graessley, W. Rheol. Acta 1977, 16, 527.

(4) Schausberger, A.; Schindlauer, G.; Janeschitz-Kriegl, H. Rheol. Acta 1985, 24, 220.

(5) Baumgaertel, M.; Schausberger, A.; Winter, H. Rheol. Acta 1990, 29,400 .

(6) Rouse, P. J. Chem. Phys. 1953, 21, 1272.

(7) Doi, M. Chem. Phys. Lett. 1974, 26 (2), 269.

(8) de Gennes, P.-G. Scaling Concepts in Polymer Physics; Cornell University Press: Ithaca, NY, 1979.

(9) Doi, M.; Edwards, S. The Theory of Polymer Dynamics; Clarendon Press: Oxford, 1986.

(10) Bird, R.; Armstrong, R.; Hassager, O.; Curtis, C. Dynamics of Polymeric Liquids, 2nd ed.; Wiley: New York, 1987.

(11) des Cloizeaux, J. Macromolecules 1990, 23, 4678.

(12) Tobolsky, A. Properties and Structures of Polymers; Wiley: New York, 1960.

(13) Ferry, J. Viscoelastic Properties of Polymers; Wiley: New York, 1980.

(14) Orbey, N.; Dealy, J. J. Rheol. 1991, 35 (6), 1035.

(15) Baumgaertel, M.; De Rosa, M.; Machado, J.; Masse, M.; Winter, H. Rheol. Acta 1992, 31, 75 .

(16) Berry, G.; Fox, T. Adv. Polym. Sci. 1968, 5, 261.

(17) Plazek, D. J. Rheol. 1992, 36 (8), 1671.

(18) Doi, M. J. Polym. Sci., Polym. Lett. Ed. 1981, 19, 265. 